\title{
Estresse, enfrentamento e sua influência sobre a glicemia e a pressão arterial
}

\author{
Stress, coping, and their influence on blood glucose and blood pressure \\ Estrés, enfrentamiento y su influencia en la glucosa y la presión arterial \\ Alex Andrade Mesquita \\ Universidade Federal do Maranhão (UFMA) \\ Centro de Referência em Saúde do Trabalhador Regional São Luís (CEREST) \\ Juliana Lima Lobato \\ Valentina Ferreira Santos de Almada Lima \\ Kheslen Pereira Brito \\ Universidade Federal do Maranhão (UFMA)
}

\begin{abstract}
Resumo
O estresse é considerado um fator que pode influenciar ou desencadear diferentes tipos de doenças, dentre elas a hipertensão arterial e a diabetes, porém o mesmo se torna crônico apenas quando as estratégias de enfrentamento do individuo falham. Os principais objetivos do estudo foram: verificar se os trabalhadores de um órgão público apresentam estresse, se existe correlação entre estresse e pressão arterial e nível glicêmico e quais as estratégias de enfrentamento utilizadas. Foi aferida a pressão arterial e medida a glicemia pós-prandial e aplicado o Inventário Lipp de Estresse Adulto e a Escala de Coping Ocupacional. Os resultados mostraram que 27,41\% dos participantes apresentam estresse. O tipo predominante de estratégia de enfrentamento foi de "controle". Não foi verificada correlação significativa entre estresse e idade, estresse e pressão arterial e estresse e nível glicêmico. Os tipos de estratégias de enfrentamento de estresse utilizados pouco influenciaram na pressão arterial e na glicemia.

Palavras-chave: Estresse; Enfrentamento; Pressão arterial; Glicemia.
\end{abstract}

\begin{abstract}
Stress is considered a factor that may influence or trigger different types of diseases, including hypertension and diabetes; however, it becomes chronic just when individuals' coping strategies fail. The main study aims were to verify whether employees of a public agency are stressed, if there is a correlation between stress and blood pressure and blood glucose level and what are the coping strategies used. Blood pressure and postprandial blood glucose were measured, and the Lipp Stress Symptom Inventory for Adults and the Occupational Coping Scale were applied. The results showed that $27.41 \%$ of the participants are affected by stress. The predominant type of coping strategy adopted was "control ". There was no significant correlation between stress and age, stress and blood pressure, or stress and glucose level. The different types of stress coping strategies used by the subjects had little influence on blood pressure and blood glucose levels.

Key-words: Stress; Coping; Blood pressure; Blood glucose.
\end{abstract}

\section{Resumen}

El estrés es considerado un factor que puede influir o desencadenar diferentes tipos de enfermedades, como la hipertensión y la diabetes, pero se vuelve crónico cuando las estrategias de enfrentamiento del individuo fallan. Los principales objetivos del estudio fueron: verificar si los trabajadores de un órgano público presentan estrés y si existe correlación entre el estrés y la presión arterial y el nivel de glucosa y cuáles son las estrategias de enfrentamiento utilizadas. Se midió la presión arterial y la glucemia postprandial. Fue aplicado el Inventário Lipp de Estrés Adulto y La Escala de Afrontamiento Ocupacional. Los resultados mostraron que 27,41 \% de los participantes presentan estrés. El tipo predominante de estrategia de enfrentamiento fue del "control". No hubo correlación significativa entre estrés y edad, estrés y presión arterial y estrés y nivel de glucosa. Los tipos de estrategias de afrontamiento de estrés utilizados tenían poca influencia en la presión arterial y la glucemia.

Palabras-clave: Estrés; Afrontamiento; Presión arterial; Glucosa.

\section{Introdução}

O estresse vem sendo atualmente considerado um dos mais importantes fatores desencadeadores de diferentes tipos de doenças na sociedade moderna. Estimativas apontam que cerca de 50\% das doenças têm alguma relação com o mesmo, seja esta direita ou indireta (Sabbatine, 1996).
De acordo com Lima (2005), dados da Academia Americana de Médicos de Família apontam que mais de $75 \%$ das consultas médicas nos Estados Unidos estão relacionadas com o estresse, o que gera um gasto de aproximadamente 150 bilhões de dólares ao ano.

Estresse pode ser definido como um estado de tensão que causa uma ruptura no equilíbrio 
interno do organismo, ou seja, um estado de tensão patogênico. O desequilíbrio ocorre quando a pessoa necessita responder a alguma demanda que ultrapassa sua capacidade adaptativa (Everly, 1990). É uma reação do organismo composta por componentes físicos e/ou psicológicos, causados pelas alterações psicofisiológicas que ocorrem quando a pessoa se depara com uma situação que, de um modo ou de outro, a irrite, amedronte, excite ou confunda, ou mesmo que a faça imensamente feliz. Isto significa que o processo bioquímico do estresse independe da causa da tensão, sendo que o elemento primordial necessário para o seu desencadeamento é claramente a necessidade de adaptação a algum fato ou mudança (Lipp, Arantes, Buriti \& Wizig, 2002). Esta resposta procura restabelecer a homeostase do organismo uma vez que ele tenha sido mobilizado para uma reação de fuga ou luta (Alchieri \& Cruz, 2008).

Selye (1956) desenvolveu o conceito de síndrome geral de adaptação (SGA) como consequência à exposição repetida e prolongada a um evento estressor. A SGA revisada por Lipp (1995) se divide em quatro fases: 1) Fase de reação ou alarme: se um estímulo estressor é forte, o organismo se prepara para uma reação de fuga ou luta, aumentando a liberação de adrenalina. Esta fase dura apenas algumas horas e a produtividade aumenta; 2) Fase de resistência: se o estressor perdura o organismo usa toda sua energia para se reequilibrar. Ocorre redução de liberação de adrenalina e aumento de corticosteróides, o organismo fica mais fraco e susceptível a doenças; 3) Fase de quase-exaustão, nesta fase inicia-se o processo de adoecimento e os órgãos que possuem maior vulnerabilidade genética ou adquirida passam a mostrar sinais de deterioração; 4) Fase de exaustão: se estressor persiste, o organismo fica exausto, podendo ocorrer distúrbios psicológicos como depressão, ansiedade aguda, vontade de fugir, dificuldade de memória, dificuldade de tomar decisões e irritabilidade. Podem ocorrer ainda, doenças como: hipertensão, gastrite, úlceras e baixa do sistema imunológico.

\section{Estresse, Pressão Arterial e Glicemia}

$\mathrm{O}$ estresse gera respostas fisiológicas incluindo atividades neurais e endócrinas, que podem influenciar os outros processos corporais, como, por exemplo, gerar aumento do funcionamento metabólico, cardiovascular e do sistema nervoso autônomo, levando ao aumento do batimento cardíaco e aumento da pressão arterial (Lima, 2005).

O sistema cardiovascular participa ativamente das adaptações ao estresse estando, portanto sujeito às influências neuro-humorais. As respostas cardiovasculares resultam principalmente em um aumento da frequência cardíaca, da contratilidade, débito cardíaco e pressão arterial. Já foi verificada a influencia do estresse na isquemia miocárdica e nas arritmias (Lourdes, Sant'Ana, Baldotto, Sousa \& Nóbrega, 2002).

Gasperin, Netuveli, Dias-da-Costa e Patussi (2009) realizaram uma revisão sistemática seguida de metanálise com o objetivo de avaliar o efeito do estresse psicológico no aumento da pressão arterial com cerca de 2043 estudos e 34.556 sujeitos. Os resultados mostraram que indivíduos com maior reação a tarefas estressoras possuíam $21 \%$ mais chances de apresentar aumento na pressão arterial quando comparados com aqueles com menor reação.

Em outro estudo Laulert, Chaves e Moura (1999) investigaram o estresse ocupacional em 207 enfermeiros de um hospital universitário e encontraram correlação entre alterações cardiovasculares e nível de estresse.

Porém, Lindquist, Beilin e Knuiman (1997) estudando 654 trabalhadores de um escritório do governo australiano não verificaram correlação significativa entre nível de estresse no trabalho e pressão arterial.

Verifica-se uma contradição nos estudos supracitados, talvez outras variáveis como o tipo de resposta de enfrentamento de estresse, idade, tipo de alimentação e padrão comportamental possam mediar ou interferir entre o estímulo estressor e as respostas de estresse, neste caso a pressão arterial.

O estresse psicossocial crônico está relacionado ao controle metabólico perturbado em adultos e crianças diabéticas (Cox, Tayor, Nowacek, HolleyWilcox \& Guthrow, 1984). Duas explicações têm sido propostas: a) $\mathrm{O}$ estresse interrompe as rotinas comportamentais e impede o gerenciamento da doença, prejudicando a alimentação, reduzindo a quantidade de exercício ou impedindo que o sujeito tome a medicação, por exemplo; b) $\mathrm{O}$ estresse inicia um processo psicofisiológico de excitação, no qual hormônios contra-reguladores são secretados, o que faz aumentar os níveis de glicose e ácidos graxos livres no sangue (Peyrot \& MacMurry, 1992).

De acordo com Sousa (2002), o estresse é uma reação fisiológica do organismo que desencadeia a liberação de hormônios e que tem por efeito a geração de energia para o corpo executar a ação de luta ou fuga. No diabetes do tipo 1 o estresse reduz os níveis de glicose, já no diabetes do tipo II, a forma mais comum da doença, aumenta os níveis de glicose. Penteado e Oliveira (2009) afirmam que ocorre a ativação continuada do eixo HPA, com hipersecreção de cortisol, entre outros hormônios, no estresse crônico. A desorganização do eixo HPA no diabetes melitus II parece envolver interações complexas entre a sensibilidade alterada à resposta hormonal e fatores como hipoinsulinemia, hiperglicemia e/ ou hipoleptinemia, estimulando o eixo HPA. Esta hiperativação está também associada com aumento do hormônio liberador de corticotrofina pelo hipotálamo 
e do receptor mineralocorticóide do hipocampo. Embora a insulina induza os níveis de ACTH e corticosterona a permanecerem dentro dos padrões de normalidade, a secreção do hormônio liberador de corticotrofina pelo hipotálamo e o receptor mineralocorticóide do hipocampo permanecem elevados, provavelmente através da supressão mediada pelo cortisol, favorecendo a mobilização de reservas de glicose e a diminuição da sensibilidade dos adipócitos à ação da insulina.

Walles (1995) considera que existe forte evidência de que o estresse psicológico está relacionado a uma deterioração do controle glicêmico em pacientes com diabetes estabelecido, porém há pouca ou nenhuma evidência de que o estresse psicológico possa produzir diabetes. Parece mais provável que o estresse psicológico produz uma deterioração da glicemia no doente não sintomático, o que por sua vez faz com que os sintomas diabéticos se tornem evidentes.

Peyrot e McMurry (1992) objetivavam verificar se os níveis de glicose de 105 pacientes diabéticos não insulino-dependentes seriam afetados pelo estresse e pelo estilo de enfrentamento de estresse utilizado. Os estilos de enfrentamento avaliados foram: Foco na resolução do problema, evitamento do problema, controle das emoções e três estilos de reação emocional (ansiedade, impaciência e raiva). Foi considerando coping efetivo quando a pessoa utilizava predominantemente um dos três primeiros estilos e coping inefetivo quando utilizava um dos três últimos. Os participantes realizaram o teste de comportamento da hemoglobina glicosada (Hba1) para mensuração da glicemia. Verificou-se que o estresse aumentou significativamente os níveis glicêmicos, porém nos indivíduos que utilizaram o enfrentamento eficiente não foi verificado aumento do nível glicêmico. Desta forma, o estilo de coping influenciou no controle glicêmico.

\section{Estresse e Enfrentamento}

O estresse ocupacional pode ser entendido como "um processo em que o indivíduo percebe demandas de trabalho como estressores, os quais, ao exceder sua habilidade de enfrentamento, provocam no sujeito reações negativas." (Way \& Carvalho, 2009, p. 564). Se o coping for adequado, o nível de estresse pode diminuir, já em caso contrário, se as estratégias de enfrentamento não forem adequadas, o nível de estresse pode se intensificar (Antoniazzi et al., 1998).

Folkman e Lazarus $(1980,1984)$ afirmam que o coping (enfrentamento) consiste em um conjunto de esforços (cognitivos e comportamentais), despendido pelos indivíduos com o objetivo de lidar com as demandas específicas (internas ou externas), que são avaliadas como excessivas em relação aos seus recursos pessoais, estando presentes em situações de estresse. Nesta direção, os autores defendem que o coping representaria a forma como as pessoas geralmente reagem ao estresse, sendo uma variável individual, determinada por fatores pessoais, exigências situacionais e recursos disponíveis.

Zautra e Wrabetz (1991) entendem o coping como um processo dinâmico de esforços despendidos para o ajustamento do organismo através da resolução das demandas exigidas, e Parkes (1994) e Terry (1994) o compreendem como um construto multidimensional, que envolve estratégias cognitivas e emocionais para alterar, reavaliar e evitar situações estressantes ou diminuir seus efeitos.

Segundo Antoniazzi, Dell'Aglio e Bandeira (1998) é importante distinguir estilo de coping de estratégias de coping. O primeiro se refere a características de personalidade ou a resultados de coping, já o segundo às ações específicas de tomada de decisão frente a um episódio estressante.

De acordo com Folkman e Lazarus (1980), as estratégias podem ser classificadas como coping focalizado na emoção e coping focalizado no problema. Nesta perspectiva, o coping focalizado na emoção seria um esforço para regular o estado emocional associado ao estresse. Desta forma, as resposta de coping são realizadas para que seja possível modificar o estado emocional do indivíduo. Já o coping focalizado no problema, seria o esforço direcionado para a modificação da situação que causou o estresse, alterando o problema existente na relação entre a pessoa e o ambiente que está causando a situação estressante.

Antoniazzi et al. (1998) destaca que o uso de qualquer uma das estratégias depende diretamente da avaliação da situação estressora feita pelo indivíduo. É importante ressaltar também que estratégias de enfrentamento com foco na emoção ou com foco no problema podem ser utilizadas ao mesmo tempo dependendo dos estressores envolvidos.

Lipp e Tanganelli (2002) investigaram o estresse ocupacional em 75 Magistrados de Justiça do Trabalho e as estratégias de enfrentamento utilizadas por eles. Os resultados apontaram que $70,6 \%$ da amostra apresentava sintomatologia de um quadro de estresse e a estratégia de enfrentamento mais utilizada por eles foi conversar com o conjugue ou com alguém com quem estejam afetivamente ligados (69\%). Pelos juízes casados, outra estratégia bastante citada foi pensar nos filhos (36\%), e pelos solteiros, sair para passear $(53 \%)$.

Sanzovo e Coelho (2007) verificaram as principais estratégias de enfrentamento de estresse utilizadas por 15 psicólogos, as mais citadas foram: fazer exercícios físicos e tirar férias.

Lindquist, Beilin e Knuiman (1997) dizem que as estratégias de enfrentamento de estresse podem ser adaptativas ou desadaptativas. No primeiro caso, por exemplo, observa-se comportamentos como: busca por apoio social, pratica exercícios físicos 
e relaxamento. No segundo caso, temos exemplos como: comer em excesso, ingerir álcool e drogas e se afastar do convívio social. O tipo de estratégia utilizada pode influenciar na saúde do indivíduo. Desta forma, variáveis como pressão arterial e nível glicêmico, podem ser alteradas.

A situação pode ser exemplificada por um caixa de banco que sofre pressão para atingir metas de vendas de seguros, títulos de capitalização e outros produtos banco, mantém contato direto com o público que muitas vezes está impaciente pela espera e tem que estar sempre atento para não cometer erros, pois caso contrário tem que pagar o dinheiro faltante. Este trabalhador pode apresentar estresse em virtude desta situação e sentir dores de estômago e aumento da pressão. Ele pode enfrentar o estresse indo a uma academia após o trabalho ou fumando e bebendo, o efeito será o mesmo no primeiro momento: se sentir mais relaxado. Mas no primeiro caso, suas dores e sua pressão podem diminuir com o passar do tempo e no segundo aumentar. E esta segunda forma de enfrentamento pode prejudicar ainda mais a saúde do indivíduo podendo deixá-lo mais estressado.

Diante do exposto, os objetivos do estudo foram: 1) Verificar se os trabalhadores de uma secretaria pública de transporte apresentam estresse e em que fase; 2) verificar quais os tipos de estratégias de enfrentamento de estresse que estes trabalhadores utilizam; 3) verificar quais as respostas comportamentais e cognitivas mais utilizadas no enfrentamento do estresse; 4) correlacionar idade e nível de estresse; 5) correlacionar estresse e pressão arterial; 6) correlacionar estresse e nível de glicemia pós-prandial e 7) verificar se indivíduos que utilizam tipos diferentes de estratégias de enfrentamento de estresse apresentam diferença nos níveis de pressão arterial e glicemia pós-prandial.

\section{Método}

\section{Participantes}

Participaram do estudo 62 funcionários da Secretaria Municipal de Trânsito e Transporte da cidade de São Luís, com idade média de 40,22 anos $(\mathrm{dp}=11,26)$, sendo 35 homens e 27 mulheres. Os cargos presentes na amostragem foram: agente de trânsito, agente administrativo, auxiliar administrativo, vigilante, serviços gerais, motorista, jornalista, secretária, copeira, supervisor de agentes, vistoriador, assessor técnico e operador de rádio. Os participantes foram selecionados por meio de voluntariado.

\section{Instrumentos}

Inventário Lipp de Estresse Adulto: O ISSL é um teste escrito validado (Lipp, 2002) composto por 23 questões de múltipla escolha que mede o grau de estresse. Ele se baseia na teoria de estresse de Seyle (1956/1970), revisada por Lipp (2000). É composto de três quadros que se referem às fases do estresse, sendo o quadro dois utilizado para avaliar as fases 2 e 3 (resistência e quase-exaustão). Os sintomas listados são típicos de cada fase. No primeiro quadro se tem 12 sintomas físicos e três psicológicos, o respondente assinala com F1 ou P1 os sintomas físicos ou psicológicos que tenha experimentado nas ultimas 24 horas. No segundo quadro, são 10 sintomas físicos e cinco psicológicos e se assinala com F2 ou P2, os sintomas experimentados na ultima semana. No quadro três, composto por 12 sintomas psicológicos e 11 físicos, assinalam-se com F3 ou P3 os sintomas experimentados no ultimo mês. Como resultado é possível se verificar em qual fase do estresse a pessoa se encontra: alerta, resistência, quase-exaustão e exaustão e a sintomatologia na área física e psicológica.

Foi utilizado por se tratar de um dos mais utilizados instrumentos no país para rastreamento do estresse, desta forma facilitando comparações com outros estudos.

Escala de Coping Ocupacional: A ECO foi desenvolvida por Latack (1986) e validada e adaptada para o português por Pinheiro, Tamayo, Tróccoli (2003), a consistência interna variou de 0,71 a 0,81 . A escala apresenta 29 estratégias cognitivas e comportamentais de enfrentamento, onde o participante deve marcar quais estratégias utiliza e sua frequência. A frequência varia de um "nunca faço isso" a cinco "sempre faço isso". Apresenta os fatores: esquiva (11 itens), controle, (nove itens) e manejo de sintomas (nove itens). Controle são ações e reavaliações proativas de enfrentamento. Esquiva consiste em ações e reavaliações cognitivas no sentido de evitar o problema. Já o manejo de sintomas são estratégias voltadas à administração dos sintomas sentidos como: relaxamento e exercício físico. Como resultado se tem qual a estratégia de enfrentamento de estresse predominantemente utilizada pelo sujeito: controle, esquiva ou manejo de sintomas.

Equipamentos

Esfigmomanômetro manual para aferição da pressão arterial.

Medidor de glicose Roche Accu-Chek Active.

\section{Procedimento}

Os participantes foram convidados a comparecerem no auditório da Secretaria Municipal de Trânsito e Transportes da cidade de São Luís, para uma "ação de saúde" onde tinham sua pressão arterial, nível glicêmico e nível de estresse avaliados. Estes preenchiam o termo de consentimento livre e esclarecido e em seguida era realizada a medição da pressão arterial e da glicemia pós-prandial. Logo após eram preenchidos os inventários ISSL e a escala ECO. Após a análise de dados foi fornecido feedback dos resultados ao órgão estudado. 
Figura 1

Porcentagem de participantes em cada fase de estresse.

$88,23 \%$

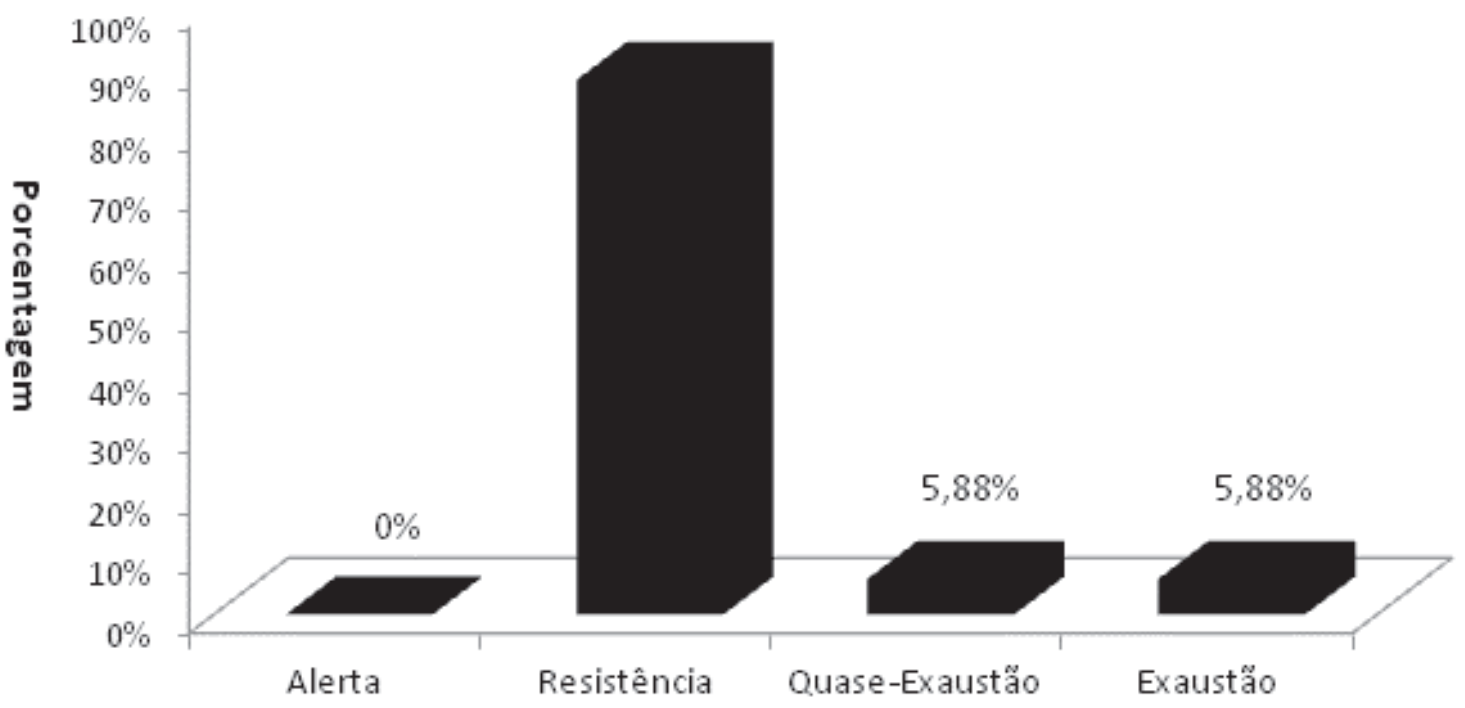

\section{Resultados}

Pode-se verificar que $27,41 \% \quad(n=17)$ dos participantes estavam estressados e $72,59 \%$ $(\mathrm{n}=45)$ não estavam estressados. Dos participantes estressados $64,7 \%(n=11)$ apresentou predominância de sintomas físicos, 29,42\% $(n=5)$ predominância de sintomas psicológicos e 5,88\% $(\mathrm{n}=1)$ predominância de sintomas físicos e psicológicos.

Em relação às fases de estresse nenhum participante estava na fase de alerta, 88,23\% $(n=15)$ estavam na fase de resistência, 5,88\% $\quad(n=1)$ na fase de quase - exaustão e $5,88 \%(\mathrm{n}=1)$ na fase de exaustão (figura 1).

A tabela 1 apresenta as correlações entre nível de estresse e idade, estresse e pressão sistólica, estresse e pressão diastólica e estresse e glicemia. Ocorreram apenas correlações positivas fracas entre as variáveis analisadas e nenhuma correlação foi significativa.

A figura 2 apresenta as estratégias de enfrentamento predominantemente utilizadas pelos sujeitos. Pode-se observar que a maioria, 64,4\%

$(n=39)$ tenta controlar as fontes de estresse, $20,34 \%$ $(n=13)$ predominantemente manejam os sintomas de estresse e $15,25 \%(n=10)$ se esquivam.

Realizou-se a média das respostas da escala ECO, para se verificar quais os tipos de comportamentos ou pensamentos mais utilizados pelos participantes para enfrentar as situações estressantes. A resposta com maior média foi: "Me esforço para fazer o que acho que se espera de mim" com média 4,26 $(\mathrm{dp}=0,85)$, seguido por "Me envolvo ainda mais nas minhas tarefas, se acho que isso pode resolver a questão" com média de 3,81 (dp=1,12) e "Tento trabalhar mais rápida e eficientemente" com média de 3,81 $(\mathrm{dp}=1,24)$. A tabela 2 apresenta os itens com maior média citados.

Para verificar se o tipo de estratégia de enfrentamento predominantemente usada para enfrentar o estresse (controle, esquiva ou manejo de sintomas) poderia influenciar nos níveis de pressão arterial e glicemia, optou-se por apresentar somente à média e o desvio padrão de cada grupo, isto em virtude do pequeno número de participantes. Pode-

Tabela 1

Correlações entre estresse e idade, estresse e pressão sistólica, estresse e pressão diastólica e estresse e glicemia

\begin{tabular}{lrc}
\hline Variáveis & Estresse & $p$ \\
\hline Idade & 0,030 & 0,818 \\
Pressão Sistólica & 0,034 & 0,801 \\
Pressão Diastólica & 0,157 & 0,240 \\
Glicemia & 0,091 & 0,501 \\
\hline
\end{tabular}


Figura 2

Estratégias de enfrentamento de estresse predominantemente utilizadas.

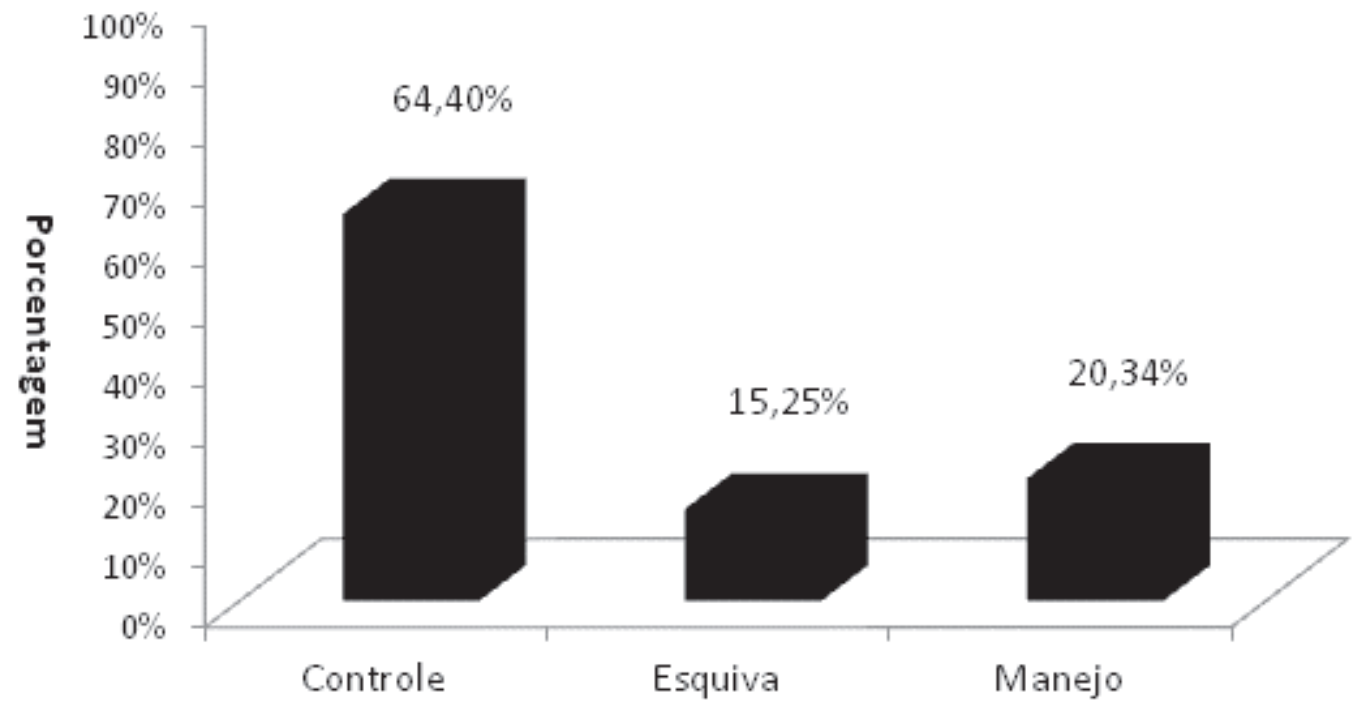

se observar apenas uma pequena diferença tanto nos níveis de pressão arterial sistólica e diastólica, quanto para o nível glicêmico nos participantes que usam estratégias de controle, esquiva ou manejo de sintomas (tabela 3).

\section{Discussão}

Foi verificado que $27,41 \%$ dos participantes estavam estressados, este percentual foi bem inferior quando comparado a outros estudos. Silva e Martinez (2005) verificaram a prevalência de estresse na população de uma cidade do interior e em uma capital, utilizando o ISSL, os resultados mostraram que $79 \%$ dos participantes estavam estressados. Comparado a estudos com servidores públicos, mesmo tipo de participante do presente estudo, como em Minari (2007) que utilizou o ISSL em 42 funcionários de um instituto público, a porcentagem de estressados foi bem mais baixa $27,41 \%$ contra $61,9 \%$. Isto pode ser visto também em relação a professores (eg. Mesquita, 2013; Júnior \& Lipp, 2008), policiais (eg. Rossetti et al., 2008) e profissionais de saúde (eg. Carvalho \& Maligris, 2007). Pode-se hipotetizar que a estabilidade do serviço público, aliada à pouca

Tabela 2

Média e desvio padrão dos itens com maior média da escala ECO

\begin{tabular}{lcc}
\hline Item & Média & $D P$ \\
\hline Me esforço para fazer o que acho que se espera de mim & 4,26 & 0,85 \\
$\begin{array}{l}\text { Me envolvo ainda mais nas minhas tarefas, se acho que } \\
\text { isso pode resolver a questão }\end{array}$ & 3,81 & 1,12 \\
Tento trabalhar mais rápida e eficientemente & 3,81 & 1,24 \\
Concentro-me em fazer prioritariamente aquilo que gosto & 3,72 & 1,15 \\
Penso na situação como um desafio & 3,64 & 1,31 \\
$\begin{array}{l}\text { Penso em mim como alguém que sempre consegue se sair } \\
\text { bem em situações como essa }\end{array}$ & 3,63 & 1,08 \\
$\begin{array}{l}\text { Tento ver a situação como uma oportunidade para aprender } \\
\text { e desenvolver novas habilidades }\end{array}$ & 3,59 & 1,33 \\
Procuro a companhia de outras pessoas & 3,53 & 1,29 \\
\hline
\end{tabular}


Tabela 3

Média e desvio padrão da pressão arterial sistólica, diastólica e nível glicêmico por grupo de estratégia de enfrentamento predominantemente utilizada.

\begin{tabular}{|c|c|c|c|c|c|c|}
\hline \multirow[t]{2}{*}{ Grupo } & \multicolumn{2}{|c|}{ Pressão Sistólica } & \multicolumn{2}{|c|}{ Pressão Diastólica } & \multicolumn{2}{|c|}{ Glicemia } \\
\hline & $M$ & $D P$ & $M$ & $D P$ & $M$ & $D P$ \\
\hline Controle & 121,14 & 16,22 & 76,67 & 10,55 & 115,63 & 23,51 \\
\hline Esquiva & 123,33 & 11,18 & 76,66 & 5,00 & 117,38 & 13,13 \\
\hline Manejo & 120,83 & 9,92 & 80,83 & 5,14 & 110,83 & 20,24 \\
\hline
\end{tabular}

pressão por resultados seja um dos fatores para que poucos participantes estivessem estressados.

Considerando todos os participantes a maior parte utiliza estratégias de enfrentamento ativas, de maneira a controlar as causas de estresse, o que pode ser considerado "saudável" ou adaptativo. Fato semelhante foi verificado por Santos e Junior (2007) que objetivaram verificar a prevalência de estresse e as estratégias de enfrentamento utilizadas por 27 mestrandos em ciências da saúde da Universidade Federal do Sergipe. Os resultados apontaram que $40,7 \%$ dos participantes apresentaram estresse e que a estratégia de enfrentamento mais utilizada pelos participantes foi "foco no problema". O mesmo ocorreu em Sousa (2005) que utilizou a escala ECO em 190 motoristas de ônibus e verificou que a maioria, $81,6 \%$ dos participantes utilizava estratégias de coping de controle predominantemente.

Apesar da relação entre hipertensão e estresse estar bem delineada (Ministério de Saúde, 2002; Sociedade Brasileira de Cardiologia, 2010; Lipp, 2008, 1996) o estudo não verificou correlação significativa entre estresse e pressão arterial e nem entre pressão arterial e tipo de estratégias de enfrentamento. O mesmo pode ser observado em Lindquist, Beilin e Knuiman (1997) estudando 654 trabalhadores de um escritório do governo australiano, que também não verificaram correlação significativa entre nível de estresse no trabalho e pressão arterial. $\mathrm{O}$ que foi encontrado é que o estilo de vida decorrente das estratégias de enfrentamento do estresse é que influenciam na pressão arterial. Estilos desadaptativos como: comportamento de consumo exagerado (eg. comida, álcool, drogas), evitamento ou negação do problema e isolamento social é que se correlacionaram à hipertensão. A presença de suporte social, atitudes positivas e prática de exercício físico foram estratégias de enfrentamento que se relacionaram com pressão arterial normal. Algumas razões para não se verificar a influencia do estresse sobre a pressão arterial no presente estudo talvez sejam: a realização de uma única medição da pressão arterial, esta pode flutuar durante o dia e também o baixo nível de estresse na amostra. Pode-se hipotetizar também que as estratégias de enfrentamento de estresse utilizadas pelos participantes podem ser adaptativas, o que reduz o nível de estresse e evita a hipertensão.
O presente estudo não verificou correlação significativa entre estresse e nível glicêmico e entre glicemia e tipo de estratégia de enfrentamento de estresse utilizada, observou-se apenas uma pequena diferença. Martinez e Latorre (2006) realizaram um estudo transversal onde foram obtidas informações sobre variáveis demográficas, ocupacionais, do estilo de vida, perfil lipídico, glicemia de jejum e pressão arterial de 3.777 empregados de uma siderúrgica. Os resultados mostraram que o estresse intenso não foi associado a alteração da pressão arterial e nem do nível glicêmico. A presente pesquisa como a de Martinez e Latorre (2006) não está em acordo com estudos como o de Peyrot e McMurry (1992) onde foi verificado que o estresse aumentou significativamente os níveis glicêmicos, porém nos indivíduos que utilizaram o enfrentamento eficiente não foi verificado aumento do nível glicêmico. Alguns motivos para este conflito nos dados nos do presente estudo e o de Peyrot e McMurry (1992) podem ser: o baixo nível de estresse da amostra do atual estudo, o tipo de teste para mensuração de glicose e o fato dos sujeitos já serem diabéticos.

Como observado o uso de estratégias de enfrentamento adequadas pode reduzir o estresse laboral e desta forma, contribuir para manutenção da saúde dos funcionários. Psicólogos e profissionais de gestão de pessoas podem realizar programas de treinamento, desenvolvimento ou psicoterapias que estimulem o uso de estratégias eficazes de controle da situação estressora como: treino assertivo, de comunicação, de resolução de problemas, de gestão de conflitos e manutenção de uma gerência democrática e com metas compartilhadas. Também, estratégias de controle da emoção como: programas coletivos de exercícios físicos, convênios com academias, recompensas por manter a saúde, ginástica laboral, momentos de descanso periódicos durante o horário de trabalho e treino de relaxamento. Estas são algumas alternativas simples e de baixo custo.

O estresse é um problema que atinge cada vez mais pessoas em virtude do estilo de vida contemporâneo, onde a individualidade, a competitividade e o excesso de atividades são socialmente valorizados e transformados em regras a serem seguidas para a obtenção do sucesso profissional e pessoal. Os participantes do atual estudo estão pouco 
estressados e quando estão, costumam enfrentá-lo de maneira a controlar suas causas, talvez por isso a maioria apresente pressão arterial e nível glicêmico normais.

\section{Referências}

Alchieri, J. C. \& Cruz, R. M. (2004). Estresse: Conceitos, métodos, medidas e possibilidades

de intervenção. São Paulo: Casa do Psicólogo.

Antoniazzi, A. S., Dell'Aglio, D. D., \& Bandeira, D. R. (1998).

O conceito de coping: uma

revisão teórica. Estudos de Psicologia (Natal), 3 (2), 273-294.

Carvalho, L., \& Malagris, L. E. N. (2007). Avaliação do nível de stress em profissionais de

saúde. Estudo e Pesquisa em Psicologia, 7 (3), 210-221.

Cox, D. J., Taylor, A. G., Nowacek, G., Holley-Wilcox, P., Pohl, S. L. \& Guthrow, E. (1984).

The relationship between psychological stress and insulin-dependent diabetic blood glucose control: preliminary investigations. Health Psychol, 3, 63-75.

Everly, G. S. (1990). A Clinical Guide to the Treatment of the Human Stress Response. New

York: Plenum Press.

Folkman, S. (1984). Personal control and stress and coping processes: a theoretical analysis. Journal of Personality and Social Psychology, 46, 839-852.

Folkman, S., \& Lazarus, R. S. (1980). An analysis of coping in a middle-aged community sample. Journal of Health and Social Behavior, 21, 219-239.

Júnior, G. E. \& Lipp, M. E. N. (2008). Estresse entre professoras do ensino fundamental de escolas públicas estaduais. Psicologia em Estudo, 13(4), 847-857.

Martinez, M. C. \& Latorre, M. R. D. O. (2006). Fatores de risco para hipertensão arterial e diabete

melito em trabalhadores de empresa metalúrgica e siderúrgica. Arquivos Brasileiros de Cardiologia, 87(4), 471-479.

Lautert, L., Chaves, E. H. B. \& Moura, G. M. S. S. (1999). O estresse na atividade gerencial do enfermeiro. Revista Panamericana de Salud Pública, 6(6), 415-425.

Lindquist, T. L., Beilin, L. J. \& Knuiman, M. W. (1997). Influence of Lifestyle, Coping, and Job Stress on Blood Pressure in Men and Women. Hypertension, 29, 1-7.

Lipp, M. E. N. \& Rocha, J. C. (2007). Pressão alta e stress: o que fazer agora? Um guia de vida para ao hipertenso. Campinas: Papirus.

Lipp, M. E .N., Arantes, J. P., Buriti, M. S. \& Witzig, T. (2002). $\mathrm{O}$ estresse em escolares. Psicologia escolar e educacional, 6(1), 5156.

Lipp, M. E. N. (2000). Manual do inventário de sintomas de stress para adultos de Lipp (ISSL). São Paulo: Casa do Psicólogo.

Lipp, M. E. N. \& Rocha, J. C. (1996). Stress, hipertensão e qualidade de vida. 2 ed. Campinas: Papirus.

Lipp, M. E. N.; Malagris, L. E. N. (1995). Manejo do Estresse, In: Range B. (org.): Psicoterapia comportamental e cognitiva: pesquisa, aplicações e problemas. Campinas: Psy.

Lipp, M. E. N. \& Tanganelli, M. S. (2002). Stress e qualidade de vida em magistrados da justiça do trabalho: diferenças entre homens e mulheres. Psicologia Reflexão e Crítica, 15(3), 537-548.

Lourdes, D. L., Sant Ana, I., Baldotto, C. S. R, Sousa, E. B. \& Nobrega, A. C. L. Estresse mental e sistema cardiovascular. Arq Bras Cardiol, 78(5), 525-30.

Mesquita, A. A,. Gomes D. S., Lobato, J. L, Godim, L. \& Souza, S. B. (no prelo). Prevalências e causas de estresse e burnout em professores. Psicologia Argumento.

Minari, M. R. T. (2007). Estresse em servidores públicos do instituto nacional de seguro social de Campo Grande- MS. Dissertação de mestrado não publicada. Universidade Católica Dom Bosco. Campo Grande, Brasil.

Ministério da Saúde (2002). Plano de reorganização da atenção à hipertensão arterial e ao diabetes mellitus. Manual de hipertensão arterial e diabetes mellitus. Brasília: Ministério da Saúde.

Parkes, K. R. (1994). Personality and coping as moderator of work stress processes: models, methods and measures. Work \& Stress, 8(2), 110-129.

Penteado, M. S. \& Oliveira, T. C. (2009). Associação estressediabetes mellitus tipo II.

Revista da Sociedade Brasileira de Clínica Médica, 7(1): 40-5. Peyrot, M. F. \& McMurry, J. F. (1992). Stress buffering and glicemic control. The Role of coping styles. Diabetes Care, 15(7), 842-846.

Pinheiro, F., Tróccoli, B. \& Tamayo, M. (2003). Mensuração de coping no ambiente ocupacional. Psicologia Teoria Pesquisa, 19 (2), 153-8.

Rossetti, M. O. (2008). O inventário de sintomas de stress para adultos de Lipp (ISSL) em servidores da polícia federal de São Paulo. Revista Brasileira de Terapia Cognitiva, 4(2), 108-120.

Sabbatni, R. M. E. (1996). Mente e doença. Disponível em:

$<$ http://www.epub.org.br/correio/medicina/corr9676.htm $>$ 1996. Acesso em: 17 mai. 2012.

Santos, A. F. \& Júnior, A. A. (2007). Estresse e estratégias de enfrentamento em mestrandos de ciências da saúde. Psicologia: Reflexão e Crítica, 20(1), 104-113.

Sanzovo, C. E. \& Coelho, M. E. C. (2007). Estressores e estratégias de coping em uma amostra de psicólogos clínicos. Estudos de Psicologia: Campinas, 24(2), 227-238.

Selye, H. (1956). The stress of life. New York: Longmans.

Silva, E. A. T. \& Martinez, A. (2005). Diferença em nível de stress em duas amostras: capital e interior do estado de São Paulo. Estudos de Psicologia, 22(1), 53-61.

Sociedade Brasileira de Cardiologia (2010). Diretrizes brasileiras de hipertensão. Revista Brasileira de Hipertensão, 17(1), 7-63.

Tamayo, M. R. \& Tróccoli, B. T. (2002). Exaustão emocional: relação com a percepção de suporte organizacional e com as estratégias de coping no trabalho. Estudos de Psicologia, 7(1), $37-$ 46.

Terry, D. (1994). Determinants of coping: the role of stable and situational factors. Journal of Personality and Social Psychology, 66, 895-910.

Wales, J.K. (1995). Does psychological stress cause diabetes? Diabetic Medicine, 12, 109-112.

Zautra, A. J. \& Wrabetz, A. B. (1991). Coping success and its relationship to psychological distress for older adults. Journal of Personality and Social Psychology, 61, 801-810. 